\title{
Synthetic X-ray emission from starburst regions
}

\author{
Laura Norci and Evert J.A. Meurs \\ Dunsink Observatory, Castleknock, Dublin 15, Ireland
}

\begin{abstract}
We have used a population synthesis programme to calculate the soft $\mathrm{X}$-ray emission from the 30 Dor cluster. Contributions from the single and binary stellar population are included. The intended future use of the programme is the simulation of extra-galactic starbursts. Nearby clusters with violent starformation activity provide suitable training grounds. Using our programme, we confirm that the stellar population of 30 Dor cannot by itself produce the totality of the observed soft X-ray emission.
\end{abstract}

\section{Introduction}

In a starburst region several components contribute to X-ray emission. Single stars emit X-rays via shocked stellar winds or coronal emission. The binary stars are stronger emitters where the X-ray radiation is produced by an accretion process from an expanding normal star to a compact companion, or by a windwind collision process. Supernova remnants are also strong soft X-ray emitters, producing X-rays from shocked material. Stars emit X-rays at various levels in essentially every phase of their lives. Both the accretion process and X-ray emission from the shocked material in a supernova explosion are instead shortlived occurrences.

\section{Programme set-up}

The soft X-ray emission from stars has been extensively studied with the help of the data sets provided by the ROSAT survey and is fairly well known. The binary stars present, on the other hand, a more difficult task. Whether in fact the accretion process occurs or not in a certain binary system and with what modalities, depends on a number of circumstances that are known with varying degrees of certainty. We have used for the evolution of the single star population the Geneva evolutionary tracks with metallicity $Z=0.008$ and normal mass loss. For the binary stars we have so far considered binaries that accrete when the primary star is either still on the MS or has evolved after this, as long as the primary's envelope remains radiative (cases $\mathrm{A} / \mathrm{Br}$ ).

\section{30 Doradus}

We have assumed for the 30 Doradus cluster (NGC 2070) a star formation history in three instantaneous bursts of increasing strength at 5, 2.5, $1 \mathrm{Myr}$ ago, an IMF 
as given by Salpeter, a stochastic star formation (Selman et al. 1999), and a total stellar mass of $5 \times 10^{4} \mathrm{M}_{\odot}$ (Kennicutt 1984). Several simulation were performed for this choice of parameters. The resulting total X-ray emission calculated by our programme, of the order of $10^{34-35} \mathrm{erg} \mathrm{s}^{-1}$, compares well with previous rough estimates based on the number of O-type stars in the cluster (Norci \& Og̈elman 1995), and confirms that the observed soft X-ray emission from 30 Dor, $\sim 10^{37} \mathrm{erg} \mathrm{s}^{-1}$, cannot entirely be accounted for by the stellar population. The X-ray luminosities quoted here are in the energy interval 0.1-2.4 keV. No SNR or high mass binary with a compact companion contributes to the X-ray emission in any of these simulations. The consistent absence of X-ray binaries is in agreement with recent Chandra observations (Portegies Zwart et al. 2002) that associate 12 of the 20 observed X-ray sources in the core of 30 Dor (R 136) with colliding wind binaries. This is at variance with earlier conclusions (Wang 1995) that suggested black-hole binaries as origin of X-ray emission for two of these sources.

Although colliding winds are not yet included in the simulations, the Chandra sources still do not account for the missing soft X-ray emission. The contribution of the hot ISM, presently not considered in our simulation, will probably account for the remaining discrepancy, as described previously by Wang \& Helfand (1991).

\section{References}

Kennicutt, R.C. 1984, ApJ 287, 116

Norci, L., Og̈elman, H. 1995, A\&A 302, 879

Portegies Zwart, S.F., Pooley, D., Lewin, W.H.G. 2002, ApJ 574, 762

Selman, F., Melnick, J., Bosch, G., Terlevich, R. 1999, A\&A 347, 532

Wang, Q.D. 1995, ApJ 453, 783

Wang, Q.D., Helfand, D.J. 1991, ApJ 370, 541 